Tôhoku Math. Journ.

30 (1978), 95-106.

\title{
VECTOR VALUED ERGODIC THEOREMS FOR A ONE-PARAMETER SEMIGROUP OF LINEAR OPERATORS
}

\author{
Shigeru Hasegawa, Ryotaro Sato and Shigeru Tsurumi
}

(Received November 6, 1976)

The purpose of this paper is to prove pointwise ergodic theorems for a one-parameter semigroup of linear operators acting on the space of functions which take their values in a reflexive Banach space and satisfying certain norm conditions.

1. Preliminaries. Let $(X, \mathscr{F}, \mu)$ be a $\sigma$-finite measure space and $(Y,|\cdot|)$ a Banach space. Let $L_{p}(X, Y)=L_{p}(X, \mathscr{F}, \mu ; Y), 1 \leqq p<+\infty$, denote the space of all strongly measurable $Y$-valued functions defined on $X$ for which the norm is given by

$$
\|f\|_{p}=\left[\int_{X}|f(x)|^{p} d \mu\right]^{1 / p}<+\infty ;
$$

and let $L_{\infty}(X, Y)=L_{\infty}(X, \mathscr{F}, \mu ; Y)$ denote the space of all strongly measurable $Y$-valued functions defined on $X$ for which the norm is given by

$$
\|f\|_{\infty}=\operatorname{ess~sup~}_{x \in X}|f(x)|<+\infty \text {. }
$$

Following Chacon [1], if $f$ is a strongly measurable function on $X$ and $a>0$, then we let

$$
\begin{aligned}
& f^{a-}(x)=[\operatorname{sgn} f(x)] \cdot \min (a,|f(x)|), \\
& f^{a+}(x)=f(x)-f^{a-}(x),
\end{aligned}
$$

where $\operatorname{sgn} f(x)=f(x) /|f(x)|$ if $f(x) \neq 0$, and $\operatorname{sgn} f(x)=0$ if $f(x)=0$.

Let $T$ be a linear operator on $L_{1}(X, Y)$ such that $\|T\|_{1} \leqq 1$ where $\|T\|_{1}=\sup \left\{\|T f\|_{1}:\|f\|_{1} \leqq 1\right\}$, and also such that, for some constant $K \geqq 1$, $\sup _{n \geq 1}\left\|T^{n} f\right\|_{\infty} \leqq K\|f\|_{\infty}$ for all $f \in L_{1}(X, Y) \cap L_{\infty}(X, Y)$. Then $T$ can be extended so that it is defined on each $L_{p}(X, Y), 1 \leqq p<+\infty$. In fact, this is done by letting for $f \in L_{p}(X, Y)$

$$
T f(x)=\lim _{a \rightarrow+0} T\left(f^{a+}\right)(x) \quad \text { a.e. on } X
$$

(see [1]). We remark that $T$ thus extended maps $L_{p}(X, Y)$ into $L_{p}(X, Y)$ with $\left\|T^{n}\right\|_{p} \leqq K$, where $\left\|T^{n}\right\|_{p}=\sup \left\{\left\|T^{n} f\right\|_{p}:\|f\|_{p} \leqq 1\right\}$. This follows from the Riesz-Thorin convexity theorem. 
Next, let $\left\{T_{t}\right\}_{0<t<+\infty}$ be a strongly continuous one-parameter semigroup of linear operators on $L_{1}(X, Y)$. This means that

(i) each $T_{t}$ is a linear operator on $L_{1}(X, Y)$,

(ii) $T_{t} T_{s}=T_{t+s}$ for all $t, s>0$,

(iii) $\lim _{t \rightarrow s}\left\|T_{t} f-T_{s} f\right\|_{1}=0$ for each $f \in L_{1}(X, Y)$ and each $s>0$.

Let us assume in addition that $\left\|T_{t}\right\|_{1} \leqq 1$ for all $t>0$ and that, for some constant $K \geqq 1, \sup _{t>0}\left\|T_{t} f\right\|_{\infty} \leqq K\|f\|_{\infty}$ for all $f \in L_{1}(X, Y) \cap$ $L_{\infty}(X, Y)$. Then, given any $p$ with $1 \leqq p<+\infty$, each $T_{t}$ is extended to a linear operator on $L_{p}(X, Y)$ with $\left\|T_{t}\right\|_{p} \leqq K$. And thus, by a standard approximation argument, $\left\{T_{t}\right\}_{0<t+\infty}$ may be regarded as a one-parameter semigroup of linear operators on $L_{p}(X, Y)$ which is strongly continuous on the interval $(0,+\infty)$ with respect to $L_{p}(X, Y)$-norm.

It is known that, given any $f \in L_{p}(X, Y)$ with $1 \leqq p<+\infty$, there exists a $Y$-valued function $g(t, x)$, defined on $(0,+\infty) \times X$ and strongly measurable with respect to the product of Lebesgue measure and $\mu$, such that, for each fixed $t>0, g(t, x)$ as a function of $x$ belongs to the equivalence class of $T_{t} f \in L_{p}(X, Y)$. In what follows, $g(t, x)$ will be denoted by $T_{t} f(x)$. Then we observe that there exists a $\mu$-null set $N(f)$, dependent on $f$ but independent of $t$, such that if $x \notin N(f)$, then the $Y$-valued function $t \mapsto T_{t} f(x)$ is Bochner integrable over every finite interval $(a, b)$ with respect to Lebesgue measure, and the integral $\int_{a}^{b} T_{t} f(x) d t$ as a function of $x$ belongs to the equivalence class of $\int_{a}^{b} T_{t} f d t \in L_{p}(X, Y)$. Similarly, we observe that there exists a $\mu$-null set $N^{\prime}(f)$, dependent on $f$ but independent of $t$, such that if $x \notin N^{\prime}(f)$, then the $Y$-valued function $t \mapsto e^{-\lambda t} T_{t} f(x)$ is Bochner integrable over the interval $(0,+\infty)$ for every $\lambda>0$, and the integral $\int_{0}^{\infty} e^{-\lambda t} T_{t} f(x) d t$ as a function of $x$ belongs to the equivalence class of $\int_{0}^{\infty} e^{-i t} T_{t} f d t \in L_{p}(X, Y)$.

2. Maximal and dominated ergodic theorems. Our investigation is based on the following slight generalization of Chacon's maximal ergodic theorem ([1], [7]).

THEOREM 1. Let $T$ be a linear operator on $L_{1}(X, Y)$ such that $\|T\|_{1} \leqq 1$ and also such that, for some constant $K \geqq 1, \sup _{n \geq 1}\left\|T^{n} f\right\|_{\infty} \leqq K\|f\|_{\infty}$ for all $f \in L_{1}(X, Y) \cap L_{\infty}(X, Y)$. If $1 \leqq p<+\infty, f \in L_{p}(X, Y)$ and $a>0$, then

$$
\int_{E(K a)}\left(a-\left|f^{a-}(x)\right|\right) d \mu \leqq \int_{X}\left|f^{a+}(x)\right| d \mu,
$$

where 


$$
E(K a)=\left\{x: \sup _{n \geq 1}\left|\frac{1}{n} \sum_{i=0}^{n-1} T^{i} f(x)\right|>K a\right\} .
$$

Proof. Since the proof of Chacon's maximal ergodic theorem [1] is easily modified to show the present theorem, we omit the details.

THEOREM 2. Let $\left\{T_{t}\right\}_{0<t<+\infty}$ be a strongly continuous one-parameter semigroup of linear operators on $L_{1}(X, Y)$ such that $\left\|T_{t}\right\|_{1} \leqq 1$ for all $t>0$ and also such that, for some constant $K \geqq 1$, $\sup _{t>0}\left\|T_{t} f\right\|_{\infty} \leqq K\|f\|_{\infty}$ for all $f \in L_{1}(X, Y) \cap L_{\infty}(X, Y)$. If $1 \leqq p<+\infty, f \in L_{p}(X, Y)$ and $a>0$, then

$$
\int_{e(K a)}\left(a-\left|f^{a-}(x)\right|\right) d \mu \leqq \int_{X}\left|f^{a+}(x)\right| d \mu
$$

where

$$
e(K a)=\left\{x: \sup _{r>0}\left|\frac{1}{r} \int_{0}^{r} T_{t} f(x) d t\right|>K a\right\} \cup\{x:|f(x)|>K a\} .
$$

Proof. This is an adaptation of the proof of Lemma VIII. 7.6 in [2]. Since, for each $x \notin N(f),(1 / r) \int_{0}^{r} T_{t} f(x) d t$ is continuous in $r$ on the interval $(0,+\infty)$, we have

$$
\sup _{r>0}\left|\frac{1}{r} \int_{0}^{r} T_{t} f(x) d t\right|=\sup _{r \in \nu}\left|\frac{1}{r} \int_{0}^{r} T_{t} f(x) d t\right| \quad(x \notin N(f)),
$$

were $D$ denotes the set of all positive rationals. Moreover, for each $r \in D$, we have

$$
\lim _{n \rightarrow+\infty}\left\|\frac{1}{r} \int_{0}^{r} T_{t} f d t-\frac{1}{r n !} \sum_{i=0}^{r n !-1}\left(T_{1 / n}\right)^{i} f\right\|_{p}=0,
$$

because $\left\{T_{t}\right\}_{0<t<+\infty}$ is strongly continuous on the interval $(0,+\infty)$ with respect to $L_{p}(X, Y)$-norm and $\left\|T_{t}\right\|_{p} \leqq K$ for all $t>0$. Thus, by the Cantor diagonal method, we can choose a subsequence $\left(n^{\prime}\right)$ of $(n)$ such that for all $r \in D$,

$$
\frac{1}{r} \int_{0}^{r} T_{t} f(x) d t=\lim _{n^{\prime} \rightarrow+\infty} \frac{1}{r n^{\prime} !} \sum_{i=0}^{r n^{\prime} !-1}\left(T_{1 / n^{\prime} !}\right)^{i} f(x) \quad \text { a.e. on } \quad X .
$$

Therefore, putting for each $n^{\prime} \geqq 1$,

$$
f_{n^{\prime}}^{*}(x)=\sup _{k \geqq 1}\left|\frac{1}{k} \sum_{i=0}^{k-1}\left(T_{1 / n^{\prime} !}\right)^{i} f(x)\right| \quad(x \in X)
$$

and

$$
E_{n^{\prime}}(K a)=\left\{x: f_{n^{\prime}}^{*}(x)>K a\right\}
$$


we have

$$
\sup _{r \in D}\left|\frac{1}{r} \int_{0}^{r} T_{t} f(x) d t\right| \leqq \liminf _{n^{\prime} \rightarrow+\infty} f_{n^{\prime}}^{*}(x) \quad \text { a.e. on } \quad X
$$

and so

$$
e(K a) \subset \liminf _{n^{\prime} \rightarrow+\infty} E_{n^{\prime}}(K a)
$$

Hence, by Theorem 1,

$$
\begin{aligned}
\int_{e(K a)}\left(a-\left|f^{a-}(x)\right|\right) d \mu & \leqq \liminf _{n^{\prime} \rightarrow+\infty} \int_{E_{n^{\prime}}(K a)}\left(a-\left|f^{a-}(x)\right|\right) d \mu \\
& \leqq \int_{X}\left|f^{a+}(x)\right| d \mu .
\end{aligned}
$$

We shall next give a dominated ergodic theorem.

THEOREM 3. Let $\left\{T_{t}\right\}_{0<t<+\infty}$ be as in Theorem 2. If $1 \leqq p<+\infty$ and $f \in L_{p}(X, Y)$, let

$$
f^{*}(x)=\sup _{r>0}\left|\frac{1}{r} \int_{0}^{r} T_{t} f(x) d t\right| \quad(x \in X) .
$$

Then $f^{*}(x)<+\infty$ for almost all $x \in X$. In paticular, if $1<p<+\infty$, then

$$
\int_{X} f^{*}(x)^{p} d \mu \leqq \frac{p}{p-1}(2 K)^{p} \int_{X}|f(x)|^{p} d \mu .
$$

Proof. By Theorem 2 we get

$$
\begin{aligned}
\frac{a}{2} \mu\left(e(K a) \backslash\left\{|f|>\frac{a}{2}\right\}\right) & \leqq \int_{e(K a)}\left(a-\left|f^{a-}(x)\right|\right) d \mu \\
& \leqq \int_{X}\left|f^{a+}(x)\right| d \mu \quad(a>0) .
\end{aligned}
$$

Hence

$$
\begin{aligned}
\mu(e(K a)) & \leqq \mu\left(\left\{|f|>\frac{a}{2}\right\}\right)+\frac{2}{a} \int_{X}\left|f^{a+}(x)\right| d \mu \\
& \leqq \frac{2}{a} \int_{\{|f|>a / 2\}}\left|f^{a-}(x)\right| d \mu+\frac{2}{a} \int_{\{|f|>a / 2\}}\left|f^{a+}(x)\right| d \mu \\
& =\frac{2}{a} \int_{\{|f|>a / 2\}}|f(x)| d \mu \quad(a>0),
\end{aligned}
$$

which implies that $f^{*}(x)<+\infty$ for almost all $x \in X$. The second assertion of the theorem is also a direct consequence of this inequality. For the 
detailed discussion, we refer the reader to the proof of Theorem VIII. 6.8 in [2].

\section{Ergodic theorems.}

THEOREM 4. Let $Y$ be a reflexive Banach space and $\left\{T_{t}\right\}_{0<t<+\infty} a$ strongly continuous one-parameter semigroup of linear operators on $L_{1}(X, Y)$ such that $\left\|T_{t}\right\|_{1} \leqq 1$ for all $t>0$ and also such that, for some constant $K \geqq 1, \sup _{t>0}\left\|T_{t} f\right\|_{\infty} \leqq K\|f\|_{\infty}$ for all $f \in L_{1}(X, Y) \cap L_{\infty}(X, Y)$. If $1 \leqq p<+\infty$ and $f \in L_{p}(X, Y)$, then the ergodic limit

$$
\lim _{r \rightarrow+\infty} \frac{1}{r} \int_{0}^{r} T_{t} f(x) d t
$$

exists for almost all $x \in X$.

Proof. We first concern ourselves with the case $1<p<+\infty$. Then the reflexivity of $Y$ implies that $L_{p}(X, Y)$ is a reflexive Banach space. Hence it is shown that the linear manifold $L$ generated by the functions of the form

$$
f=h+\left(g-T_{s} g\right),
$$

where $h, g \in L_{p}(X, Y) \cap L_{\infty}(X, Y)$ and $T_{t} h=h$ for all $t>0$, is dense in $L_{p}(X, Y)$. (This is due to Kakutani and Yosida. See, e.g., Corollary VIII. 7.2 in [2].) We notice that if $f \in L$ then the ergodic limit in the theorem exists for almost all $x \in X$. In fact, this follows from considering the case $f=g-T_{s} g$ where $g \in L_{p}(X, Y) \cap L_{\infty}(X, Y)$. If this is the case, then we get

$$
\begin{aligned}
\left|\frac{1}{r} \int_{0}^{r} T_{t} f(x) d t\right| & \leqq \frac{1}{r}\left(\int_{0}^{s}\left|T_{t} g(x)\right| d t+\int_{r}^{r+s}\left|T_{t} g(x)\right| d t\right) \\
& \leqq \frac{2 s}{r} K\|g\|_{\infty} \rightarrow 0 \quad \text { a.e. on } X
\end{aligned}
$$

as $r \rightarrow+\infty$, and hence $\lim _{r \rightarrow+\infty}(1 / r) \int_{0}^{r} T_{t} f(x) d t=0$ a.e. on $X$.

Now assume that $f$ is an arbitrary function in $L_{p}(X, Y)$. Choose a sequence $\left(f_{n}\right)$ of functions in $L$ satisfying $\lim _{n \rightarrow+\infty}\left\|f-f_{n}\right\|_{p}=0$ and put $g_{n}=f-f_{n}$ and

$$
g_{n}^{*}(x)=\sup _{r>0}\left|\frac{1}{r} \int_{0}^{r} T_{t} g_{n}(x) d t\right| \quad(x \in X) .
$$

Then we get, by Theorem 3, that

$$
\int_{X} g_{n}^{*}(x)^{p} d \mu \leqq \frac{p}{p-1}(2 K)^{p}\left\|g_{n}\right\|_{p}^{p} \rightarrow 0
$$


as $n \rightarrow+\infty$. Therefore we may write

$$
\frac{1}{r} \int_{0}^{r} T_{t} f(x) d t=\frac{1}{r} \int_{0}^{r} T_{t} f_{n}(x) d t+\frac{1}{r} \int_{0}^{r} T_{t} g_{n}(x) d t,
$$

where $\lim _{r \rightarrow+\infty}(1 / r) \int_{0}^{r} T_{t} f_{n}(x) d t$ exists for almost all $x \in X$, for all $n \geqq 1$, and where, for each $\varepsilon>0,0 \leqq g_{n}^{*}(x)<\varepsilon$ except on a set of measure less than $\varepsilon$, for each sufficiently large $n$.

This proves the theorem for the case $1<p<+\infty$.

We next concern ourselves with the case $p=1$. In this case, since $L_{1}(X, Y) \cap L_{2}(X, Y)$ is dense in $L_{1}(X, Y)$, the above argument shows that it is enough to notice that if $\left(f_{n}\right)$ is a sequence of functions in $L_{1}(X, Y)$ satisfying $\lim _{n \rightarrow+\infty}\left\|f_{n}\right\|_{1}=0$, then we have

$$
\lim _{n \rightarrow+\infty} \mu\left(\left\{x: f_{n}^{*}(x)>K a\right\}\right) \leqq \lim _{n \rightarrow+\infty} \frac{2}{a}\left\|f_{n}\right\|_{1}=0 \quad(a>0),
$$

where

$$
f_{n}^{*}(x)=\sup _{r>0}\left|\frac{1}{r} \int_{0}^{r} T_{t} f_{n}(x) d t\right| \quad(x \in X) .
$$

This inequality is an immediate consequence of Theorem 2 as observed in the proof of Theorem 3, and the proof is completed.

TheOREM 5. Let $Y$ and $\left\{T_{t}\right\}_{0<t<+\infty}$ be as in Theorem 4 . If $1 \leqq p<+\infty$ and $f \in L_{p}(X, Y)$, then the local ergodic limit

$$
\lim _{r \rightarrow+0} \frac{1}{r} \int_{0}^{r} T_{t} f(x) d t
$$

exists for almost all $x \in X$.

It should be remarked here that this theorem and the next theorem have been obtained by one of the authors if $Y$ is the field of complex numbers and $K=1$ ([6]); for another related result we refer the reader to [4].

Proof. We first concern ourselves with the case $1<p<+\infty$. Then, since $L_{p}(X, Y)$ is a reflexive Banach space and $\left\|T_{t}\right\|_{p} \leqq K$ for all $t>0$, it follows that, for each $f \in L_{p}(X, Y)$, the set $\left\{T_{t} f: t>0\right\}$ is weakly sequentially compact in $L_{p}(X, Y)$. Using this fact we shall prove that $T_{t}$ on $L_{p}(X, Y)$ converges strongly as $t \rightarrow+0$.

To do this, we notice that

$$
\left\{f \in L_{p}(X, Y): \lim _{t \rightarrow+0}\left\|T_{t} f-f\right\|_{p}=0\right\}=\bigcup_{t>0} T_{t} L_{p}(X, Y),
$$


and that for each $f \in L_{p}(X, Y)$ there exists a closed separable subspace $B(f)$ of $L_{p}(X, Y)$ such that $f \in B(f)$ and $T_{t} B(f) \subset B(f)$ for all $t>0$. Let $\left\{f_{i}: i \geqq 1\right\}$ be a dense subset of $B(f)$. Then by the Cantor diagonal method, we can find a sequence $\left(t_{n}\right)$ of positive reals, with $\lim _{n \rightarrow+\infty} t_{n}=0$, such that weak- $\lim _{n \rightarrow+\infty} T_{t_{n}} f_{i}$ exists for all $f_{i}$. It follows by a standard approximation argument that weak- $\lim _{n \rightarrow+\infty} T_{t_{n}} h$ exists for all $h \in B(f)$.

If we define

$$
T_{0} h=\text { weak- } \lim _{n \rightarrow+\infty} T_{t_{n}} h \quad(h \in B(f)),
$$

then it is easily shown that $T_{0}$ is a linear operator on $B(f)$ with $\left\|T_{0}\right\|_{p} \leqq K$ and $T_{t} T_{0}=T_{t}=T_{0} T_{t}$ on $B(f)$ for all $t \geqq 0$. Hence $f$ can be written as $f=f_{1}+f_{2}$, where $T_{0} f=f_{1}$ and $T_{\mathrm{r}} f_{2}=0$. Since $f_{1}=T_{0} f_{1}=$ weak- $\lim _{n \rightarrow+\infty} T_{t_{n}} f_{1}$, it follows that $f_{3} \in \overline{\mathrm{U}}_{t>0} T_{t} L_{p}(X, Y)$. Therefore we conclude that

$$
\lim _{t \rightarrow+0}\left\|T_{t} f-f_{1}\right\|_{p}=\lim _{t \rightarrow+0}\left\|T_{t} f_{1}-f_{1}\right\|_{p}=0 \text {. }
$$

This proves that $T_{t}$ on $L_{p}(X, Y)$ converges strongly as $t \rightarrow+0$. (Here we note that this strong convergence is treated in [5] under a more general setting.)

Let us write $T_{0}=$ strong- $\lim _{t \rightarrow+0} T_{t}$. Then we have that, for each $f \in L_{p}(X, Y), \lim _{t \rightarrow+0}\left\|T_{t} f-T_{0} f\right\|_{p}=0$ and $T_{t}\left(f-T_{0} f\right)=0$ for all $t \geqq 0$. Hence it follows that

$$
\left\|\frac{1}{a} \int_{0}^{a} T_{t} f d t-T_{0} f\right\|_{p} \leqq \frac{1}{a} \int_{0}^{a}\left\|T_{t} f-T_{0} f\right\|_{p} d t \rightarrow 0
$$

as $a \rightarrow+0$, and therefore the set $M$ of the functions of the form

$$
f=\frac{1}{a} \int_{0}^{a} T_{t} g d t+h,
$$

where $g, h \in L_{p}(X, Y)$ and $T_{t} h=0$ for all $t \geqq 0$, is dense in $L_{p}(X, Y)$.

We shall prove that if $f \in M$ then $\lim _{r \rightarrow+0}(1 / r) \int_{0}^{r} T_{t} f(x) d t$ exists for almost all $x \in X$. For this purpose it is enough to consider the case $f=\int_{0}^{a} T_{t} g d t$, where $g \in L_{p}(X, Y)$. If this is the case, define a $Y$-valued function $\xi(t, x)$ on $(0,+\infty) \times X$ by the relation

$$
\xi(t, x)=\int_{t}^{t+a} T_{s} g(x) d s \quad((t, x) \in(0,+\infty) \times X) .
$$

Then it is easily seen that $\xi(t, x)$ is a strongly measurable function with respect to the product of Lebesgue measure and $\mu$, and that, for each fixed $t>0, \xi(t, x)$ as a function of $x$ belongs to the equivalence class of 
$T_{t} f \in L_{p}(X, Y)$. Moreover, for almost all $x \in X$, we have

$$
\lim _{t \rightarrow+0} \xi(t, x)=\int_{0}^{a} T_{s} g(x) d s .
$$

Therefore

$$
\begin{array}{r}
\lim _{r \rightarrow+0} \frac{1}{r} \int_{0}^{r} T_{t} f(x) d t=\lim _{r \rightarrow+0} \frac{1}{r} \int_{0}^{r} \xi(t, x) d t \\
=\lim _{t \rightarrow+0} \xi(t, x)=\int_{0}^{a} T_{s} g(x) d s=f(x)
\end{array}
$$

for almost all $x \in X$.

Now assume that $f$ is an arbitrary function in $L_{p}(X, Y)$. Choose a sequence $\left(f_{n}\right)$ of functions in $M$ satisfying $\lim _{n \rightarrow+\infty}\left\|f-f_{n}\right\|_{p}=0$, and put $g_{n}=f-f_{n}$ and

$$
g_{n}^{*}(x)=\sup _{r>0}\left|\frac{1}{r} \int_{0}^{r} T_{t} g_{n}(x) d t\right| \quad(x \in X) .
$$

Then we may write, as in the proof of Theorem 4, that

$$
\frac{1}{r} \int_{0}^{r} T_{t} f(x) d t=\frac{1}{r} \int_{0}^{r} T_{t} f_{n}(x) d t+\frac{1}{r} \int_{0}^{r} T_{t} g_{n}(x) d t,
$$

where $\lim _{r \rightarrow+0}(1 / r) \int_{0}^{r} T_{t} f_{n}(x) d t$ exists for almost all $x \in X$, for all $n \geqq 1$, and where, for each $\varepsilon>0,0 \leqq g_{n}^{*}(x)<\varepsilon$ except on a set of measure less than $\varepsilon$, for each sufficiently large $n$.

This proves the theorem for the case $1<p<+\infty$.

The proof for the case $p=1$ is exactly the same as the corresponding part of the proof of Theorem 4, and we omit the details.

THEOREM 6. Let $Y$ and $\left\{T_{t}\right\}_{0<t<+\infty}$ be as in Theorem 4 and put, for all $f \in L_{1}(X, Y)$,

$$
T_{0} f(x)=\lim _{r \rightarrow+0} \frac{1}{r} \int_{0}^{r} T_{t} f(x) d t \quad \text { a.e. on } X .
$$

Then $T_{0}$ is a linear operator on $L_{1}(X, Y)$ with $\left\|T_{0}\right\|_{1} \leqq 1$ and $\left\|T_{0} f\right\|_{\infty} \leqq$ $K\|f\|_{\infty}$ for all $f \in L_{1}(X, Y) \cap L_{\infty}(X, Y)$, and further

$$
\lim _{t \rightarrow+0}\left\|T_{t} f-T_{0} f\right\|_{1}=0
$$

for all $f \in L_{1}(X, Y)$.

Proof. It is obvious that the operator $T_{0}$ is linear and satisfies $\left\|T_{0}\right\|_{1} \leqq 1$ and $\left\|T_{0} f\right\|_{\infty} \leqq K\|f\|_{\infty}$ for all $f \in L_{1}(X, Y) \cap L_{\infty}(X, Y)$. It also follows from the proof of Theorem 5 that, on each $L_{p}(X, Y)$ with $1<$ 
$p<+\infty, T_{0}=$ strong- $\lim _{t \rightarrow+0} T_{t}$ and $T_{t} T_{0}=T_{0} T_{t}=T_{t}$ for all $t \geqq 0$. Hence, by a standard approximation argument, we get that, on $L_{1}(X, Y), T_{t} T_{0}=$ $T_{0} T_{t}=T_{t}$ for all $t \geqq 0$.

Let $f$ be an arbitrary function in $L_{1}(X, Y)$, and write $f_{0}=T_{0} f$. Then, since $T_{t} f=T_{t} f_{0}$ for all $t \geqq 0$, it suffices to show that

$$
\lim _{t \rightarrow+0}\left\|T_{t} f_{0}-f_{0}\right\|_{1}=0 \text {. }
$$

To prove this, we first notice that

$$
f_{0}(x)=\lim _{r \rightarrow+0} \frac{1}{r} \int_{0}^{r} T_{t} f_{0}(x) d t \quad \text { a.e. on } X \text {, }
$$

because $T_{0} f_{0}=f_{0}$. Hence, by Fatou's lemma, we get

$$
\left\|f_{0}\right\|_{1} \leqq \liminf _{r \rightarrow+0}\left\|\frac{1}{r} \int_{0}^{r} T_{t} f_{0} d t\right\|_{1} .
$$

Then, since $\left\|T_{t}\right\|_{1} \leqq 1$ for all $t \geqq 0$,

$$
\left\|f_{0}\right\|_{1}=\lim _{r \rightarrow+0}\left\|\frac{1}{r} \int_{0}^{r} T_{t} f_{0} d t\right\|_{1} .
$$

Thus we obtain

$$
\lim _{r \rightarrow+0}\left\|f_{0}-\frac{1}{r} \int_{0}^{r} T_{t} f_{0} d t\right\|_{1}=0 .
$$

Hence $f_{0}$ belongs to the closed linear manifald generated by the set $\left\{T_{t} f_{0}: t>0\right\}$. Since $\left\|T_{t}\right\|_{1} \leqq 1$ for all $t>0$, this together with an easy approximation argument shows that

$$
\lim _{t \rightarrow+0}\left\|T_{t} f_{0}-f_{0}\right\|_{1}=0 \text {. }
$$

This completes the proof.

\section{Abelian ergodic theorems.}

THEOREM 7. Let $Y$ be a reflexive Banach space and $\left\{T_{t}\right\}_{0<t<+\infty} a$ strongly continuous one-parameter semigroup of linear operators on $L_{1}(X, Y)$ such that $\left\|T_{t}\right\|_{1} \leqq 1$ for all $t>0$ and also such that, for some constant $K \geqq 1, \sup _{t>0}\left\|T_{t} f\right\|_{\infty} \leqq K\|f\|_{\infty}$ for all $f \in L_{1}(X, Y) \cap L_{\infty}(X, Y)$. If $1 \leqq p<+\infty$ and $f \in L_{p}(X, Y)$, then the abelian ergodic limit

$$
\lim _{\lambda \rightarrow+0} \lambda \int_{0}^{\infty} e^{-\lambda t} T_{t} f(x) d t
$$

exists and coincides with the ergodic limit in Theorem 4 for almost all $x \in X$. 
Proof. Let $f$ be an arbitrary function in $L_{p}(X, Y)$ where $1 \leqq p<$ $+\infty$. Since there exists a $\mu$-null set $N^{\prime}(f)$, dependent on $f$ but independent of $t$, such that if $x \notin N^{\prime}(f)$ then

$$
\int_{0}^{\infty} e^{-\lambda t}\left|T_{t} f(x)\right| d t<+\infty \quad(\lambda>0) .
$$

We have that, for $x \notin N^{\prime}(f)$ and $\lambda>0$,

$$
\lambda \int_{0}^{\infty} e^{-\lambda t} T_{t} f(x) d t=\lambda^{2} \int_{0}^{\infty} t e^{-\lambda t}\left[\frac{1}{t} \int_{0}^{t} T_{s} f(x) d s\right] d t
$$

and that

$$
\lambda^{2} \int_{0}^{\infty} t e^{-\lambda t} d t=1
$$

Now let $\widetilde{f}(x)$ denote the ergodic limit in Theorem 4 , and let $f^{*}(x)$ be defined as in Theorem 3. Then

$$
\begin{aligned}
& \left|\lambda \int_{0}^{\infty} e^{-\lambda t} T_{t} f(x) d t-\tilde{f}(x)\right| \\
& \quad=\left|\lambda^{2} \int_{0}^{\infty} t e^{-\lambda t}\left[\frac{1}{t} \int_{0}^{t} T_{s} f(x) d s-\widetilde{f}(x)\right] d t\right| \\
& \quad \leqq 2 f^{*}(x) \cdot \lambda^{2} \int_{0}^{a} t e^{-\lambda t} d t+\sup _{t \geqq a}\left|\frac{1}{t} \int_{0}^{t} T_{s} f(x) d s-\widetilde{f}(x)\right| .
\end{aligned}
$$

Hence, letting $\lambda \rightarrow+0$ and then $a \rightarrow+\infty$, we get the conclusion.

THEOREM 8. Let $Y$ and $\left\{T_{t}\right\}_{0<t<+\infty}$ be as in Theorem 7. If $1 \leqq p<+\infty$ and $f \in L_{p}(X, Y)$, then the local abelian ergodic limit

$$
\lim _{\lambda \rightarrow+\infty} \lambda \int_{0}^{\infty} e^{-\lambda t} T_{t} f(x) d t
$$

exists and coincides with the local ergodic limit in Theorem 5 for almost all $x \in X$.

Proof. Using Theorems 5 and 3, the theorem is shown by the same argument as in the proof of Theorem 7, and so we omit the details.

5. Remarks on the discrete case. Using Theorem 1 and modifying the proof of Theorem 4 we can prove the following slight generalization of Chacon's ergodic theorem ([1], [7]).

THEOREM 9. Let $Y$ be a reflexive Banach space and $T$ a linear operator on $L_{1}(X, Y)$ such that $\|T\|_{1} \leqq 1$ and also such that, for some constant $K \geqq 1, \sup _{n \geqq 1}\left\|T^{n} f\right\|_{\infty} \leqq K\|f\|_{\infty}$ for all $f \in L_{1}(X, Y) \cap L_{\infty}(X, Y)$. If $1 \leqq p<+\infty$ and $f \in L_{p}(X, Y)$, then the ergodic limit 


$$
\lim _{n \rightarrow+\infty} \frac{1}{n} \sum_{i=0}^{n-1} T^{i} f(x)
$$

exists for almost all $x \in X$.

Now, using Theorem 9 and modifying the proof of Theorem 7, we shall prove the following abelian ergodic theorem.

THEOREM 10. Let $Y$ and $T$ be as in Theorem 9. If $1 \leqq p<+\infty$ and $f \in L_{p}(X, Y)$, then the abelian ergodic limit

$$
\lim _{\lambda \rightarrow 1-0}(1-\lambda) \sum_{n=0}^{\infty} \lambda^{n} T^{n} f(x)
$$

exists and coincides with the limit in Theorem 9 for almost all $x \in X$.

Here we note that the existence of the abelian ergodic limits in Theorems 10 and 7 was proved by Kopp [3]. However it should seem that the arguments in the proof of Theorem 1 in [3] must be suitably corrected.

Proof. Let $f$ be an arbitrary function in $L_{p}(X, Y)$, where $1 \leqq p<+\infty$. Since $\left\|T^{n}\right\|_{p} \leqq K$ for all $n \geqq 0$, it follows that there exists a $\mu$-null set $N$, dependent on $f$ but independent of $n$, such that if $x \notin N$ then

$$
\sum_{n=0}^{\infty} \lambda^{n}\left|T^{n} f(x)\right|<+\infty \quad \text { for all } \lambda(0<\lambda<1)
$$

Hence, for all $x \notin N$, we have that

$$
\begin{aligned}
(1-\lambda) \sum_{n=0}^{\infty} \lambda^{n} T^{n} f(x) & =(1-\lambda)^{2} \sum_{n=0}^{\infty}\left[\lambda^{n} \sum_{i=0}^{n} T^{i} f(x)\right] \\
& =(1-\lambda)^{2} \sum_{n=0}^{\infty}\left[(n+1) \lambda^{n}\left(\frac{1}{n+1} \sum_{i=0}^{n} T^{i} f(x)\right)\right], \\
(1-\lambda)^{2} \sum_{n=0}^{\infty}(n+1) \lambda^{n} & =1 .
\end{aligned}
$$

Thus, using Theorem 9, the theorem is proved similarly as Theorem 7.

\section{REFERENCES}

[1] R. V. Chacon, An ergodic theorem for operators satisfying norm conditions, J. Math. Mech., 11 (1962), 165-172.

[2] N. Dunford and J. T. Schwartz, Linear Operators, Part I, Interscience, New York, 1958.

[3] P. E. KopP, Abelian ergodic theorems for vector-valued functions, Glasgow Math. J., 16 (1975), 57-60.

[4] R. Sato, Local ergodic properties of $L_{p^{-}}$operator semigroups, Comment. Math. Univ. Carolinae, 14 (1973), 177-181. 
[5] R. SATo, A note on a local ergodic theorem, Comment. Math. Univ. Carolinae, 16 (1975), 1-11.

[6] R. Sato, On a local ergodic theorem, Studia Math., 58 (1976), 1-5.

[7] L. Sucheston, Problems, in Probability in Banach Spaces (Proc. First Internat. Conferrence on Probability in Banach Spaces, Oberwolfach, 1975), 285-290, Springer, Berlin-Heidelberg-New York, 1976.

Department of Mathematics, Shibaura Institute of Technology

OMixa, SaItama, Japan

Department of Mathematics, Josai University

SAITAMA, JAPAN

Department of Mathematics, Tokyo Metropolitan University

TOKYO, JAPAN 